\title{
FIDDLE: Efficient Assembly of Networks That Satisfy Desired Behavior
}

\author{
Adam A. Butchy \\ University of Pittsburgh \\ Cheryl A. Telmer \\ Carnegie Mellon University \\ Natasa Miskov-Zivanov ( $\nabla$ nmzivanov@pitt.edu ) \\ University of Pittsburgh
}

\section{Research Article}

Keywords: Automatic Model Creation, Biological Networks, Extending Biological Networks, Model Construction, Network Reconstruction

Posted Date: June 2nd, 2021

DOl: https://doi.org/10.21203/rs.3.rs-562692/v1

License: (c) (i) This work is licensed under a Creative Commons Attribution 4.0 International License. Read Full License 
3 Adam A. Butchy ${ }^{1}$, Cheryl A. Telmer ${ }^{2}$, and Natasa Miskov-Zivanov ${ }^{1,3^{*}}$

4

5

6

$9 \quad{ }^{1}$ Department of Bioengineering, University of Pittsburgh, Pittsburgh, PA, USA

10 (adam.butchy@pitt.edu)

$11{ }^{2}$ Molecular Biosensor and Imaging Center, Carnegie Mellon University, Pittsburgh, PA, USA $12 \quad$ (ctelmer@cmu.edu)

$13{ }^{3}$ Department of Electrical and Computer Engineering, Department of Computational Biology,

14 University of Pittsburgh, Pittsburgh, PA, USA

15 (nmzivanov@ pitt.edu)

$16 *$ Corresponding Author 


\section{Abstract:}

2 Background: Due to the complexity and redundancy of biological systems, computational

3 models are difficult and laborious to create and update. Therefore, machine reading and

4 automated model assembly are of great interest to computational and systems biologists. Here,

5 we describe FIDDLE (Finding Interactions using Diagram Driven modeL Extension), the tool

6 that we built with the goal to automatically assemble or extend models with the knowledge

7 extracted from published literature. The two main methods developed as part of FIDDLE are

8 called Breadth First Addition (BFA) and Depth First Addition (DFA), and they are based on

9 network search algorithms. To assess the advantages and limitations of BFA and DFA, we

10 applied them on Erdös-Rényi random networks (ER) and Barabási-Albert scale-free networks

11 (BA) as models of biological networks. Starting with several different baseline models and

12 different sets of candidate edges, we conducted a comprehensive evaluation of the BFA and

13 DFA algorithms and their ability to either reconstruct a specified network structure or assemble

14 models that reproduce the same behavior as defined golden models.

15 Results: When BFA and DFA are applied to random networks, we are able to show that model

16 extension with BFA is more specific and DFA is a more sensitive approach. When applied to

17 scale-free networks, both BFA and DFA demonstrated very limited success.

18 Conclusion: Results suggest that BFA is a better method for assembling abundant but mixed

19 true and false information, while DFA will better assemble fewer and more truthful interactions.

20 The limited success with scale-free networks suggests a fundamental difficulty with

21 automatically creating biological models due to the inherent redundancy and complexity of

22 biological networks. These methods represent an early attempt and a novel approach to truly 
1 autonomous model creation or extension given different types of knowledge. The source code is

2 available at: https://bitbucket.org/biodesignlab/fiddle/.

4 Keywords: Automatic Model Creation/Biological Networks/Extending Biological

5 Networks/Model Construction/Network Reconstruction

\section{$7 \quad$ Background}

8 Computational approaches to modeling large complex systems standardize the representation of

9 knowledge, while simulation of computational models illuminates the dynamics of systems,

10 allowing for discoveries and theoretical advances (1). Modeling of physical and engineered

11 systems has led to insights ranging from explanations of fundamental principles to applications

12 such as robust flight simulators.

13 However, biological systems have proven difficult to model, in part due to their complexity and

14 robustness to failure and noise. There are two main approaches to modeling these systems,

15 bottom-up and top-down (2). In a bottom-up approach, known molecular interactions are

16 assembled into a model to help explain the system's behavior and predict how the system will

17 respond to new stimuli or inputs. This method has been used extensively by biologists,

18 biochemists, and molecular biologists to manually create models based on the interactions that

19 are supported in scientific literature. In a top-down approach, experimental data—usually

20 collected with high-throughput methods of data collection — is used to infer relationships

21 between elements and determine causal relationships. Top-down approaches employ many

22 different methods such as Bayesian Inference (3), ANOVA calculations (4), and Fuzzy Logic (5)

23 among others. In both the mechanistic bottom-up approach and the data-driven top-down 
1 approach, the model is used to predict the behavior of individual elements in the network $(6,7)$.

2 Recently, there has been a push to integrate the two methods, using experimental data to

3 contextualize the bottom-up approach, and incorporating prior knowledge into the top-down

4 approach to reduce the number of potential models (8-12). Despite these efforts, modeling

5 biological systems remains a tedious, iterative, and ongoing pursuit. Thus, useful models are

6 rarely built for large systems and even more rarely updated to incorporate new information. This

7 laborious process results in slow model development and few model extensions. It is because of

8 these factors, that system and computational biologists have endeavored to automate the process

9 of model creation and extension.

11 In the advent of Big Data, more information is available than ever before about molecular

12 interactions. To automatically create models, information can be extracted from literature,

13 queried from databases, or taken from existing baseline models. Public databases such as

14 Reactome (13), MetaCyc (14), OmniPath (15), and STRING (16) offer easy access to millions of

15 interactions. Additionally, there exist a number of model databases with published models that

16 are publicly available such as The Nature Pathway Interaction Database (17), WikiPathways

17 (18), BioModels (19), the Cell Collective (20), and KEGG pathways (21). These databases

18 contain highly targeted, curated published and unpublished models which are created for specific

19 biological context and may not be generalizable to explain other phenomena. When new

20 interactions are discovered, and described in a scientific publication, state-of-the-art machine

21 reading engines such as REACH (22), TRIPS (23), EVEX (24), and Rubicon (25) can extract

22 them, together with other relevant information. These automated readers are able to extract tens

23 of thousands of biological entity interactions from hundreds of papers in a few hours, and 
1 produce a machine-readable, structured output (22). Many of the interactions which reading

2 engines extract are known or incorrect, with only a few exceptions being true, novel interaction

3 which are not yet in curated databases. However, despite this abundance of available

4 interactions, there is still no efficient way to assemble them into reliable models.

6 Existing methods for collecting biological interactions, assembling a model, and performing

7 simulations include Path2Models (26) and INDRA $(27,28)$, which automatically generate both

8 quantitative and qualitative models from available pathway information. Both methods can

9 assemble models based on the quality and granularity of the information they are given;

10 however, truly representative models are dependent upon the modeling approach and context,

11 which in turn depends on human oversight, intervention and correctness of starting information.

12 These techniques rely on accurate information, and their performance suffers when the

13 interaction information is inaccurate, incomplete, or from a different biological context.

14 Recently, several other methods have been proposed that combine simulations with formal

15 model analysis (29) to automatically expand, test and select the best model, with respect to a

16 given performance metric. These new tools include the Markov clustering approach (30) and

17 genetic algorithm-based model extension (31). These approaches have different strengths and

18 weaknesses. The Markov clustering approach to model extension is well suited for the

19 combinatorial explosion in numbers of possible extensions while the genetic algorithm approach

20 is overwhelmed by large numbers of extensions. Markov clustering prioritizes strongly

21 connected components at the expense of interactions involving nodes of low degree. The genetic

22 algorithm explores the effect of single extensions distributed throughout the network. 
1 Here, we propose FIDDLE (Finding Interactions using Diagram Driven modeL Extension), a

2 tool that conducts model assembly in a bottom-up approach. The goal of FIDDLE is to assemble

3 a model that satisfies a given performance metric (defined formally in Methods), starting from a

4 list of known biological interactions, and key indicators of the studied biological phenomena. We

5 use the key indicators as ground truths when computing the error in our model performance

6 metric. FIDDLE is able to refine models by systematically adding known biological interactions

7 into intermediate models, measuring changes in model performance, and then adding or

8 discarding interactions based on whether they improve the model performance metric. FIDDLE

9 incorporates two novel algorithms, Breadth First Addition (BFA) and Depth First Addition

10 (DFA), which utilize the same principles as the breadth-first search and depth-first search

11 algorithms in network studies (32). These methods not only represent a new approach to bottom-

12 up model assembly but are also used to demonstrate the existence of key biological properties

13 which hinder automated modeling of biological systems. The model structure can be illustrated

14 using networks, where model elements (such as proteins, genes, biological processes, etc.) are

15 represented by nodes, and the molecular interactions (such as activation, inhibition, etc.) are

16 represented by directed edges. Including the direction in element connections allows for the

17 representation of causal relationships between nodes, and consequently, enables studies of the

18 complex system dynamics that arises from these causal relationships. We show a high-level

19 overview of FIDDLE in Figure 1.

21 Figure 1: "Overview of the FIDDLE process" FIDDLE is a robust model assembly tool

22 which requires candidate edges, an expectation of model behavior, and an optional baseline 
1 model. FIDDLE iteratively assembles candidate edges into candidate models, simulates

2 them, and calculates their performance using the TME metric.

4 To evaluate FIDDLE and the proposed BFA and DFA algorithms, we created a number of

5 artificial networks, systematically removed network edges, and tasked FIDDLE to re-build the

6 original model. We used artificial networks so that the structure and dynamics of the original

7 network were completely known, and we could vary key parameters in their creation to observe

8 the impact it would have on reassembly. We created two different types of networks: Erdös-

9 Rényi random networks (ER) (33) and Barabási-Albert scale-free networks (BA) (34). Biological

10 models, power grids and the internet follow scale-free power distributions. We will evaluate the

11 two network types to examine the role of network structure and determine the algorithmic

12 performance by comparing the behavior of the original and the reconstructed model.

14 We also demonstrate the advantages to using our BFA and DFA algorithms for automated model

15 assembly. Throughout our analysis, we contrast the differences between them, illustrating their

16 relative strengths, weaknesses and commonalities. By using different network structures and

17 different types of candidate knowledge, we are able to gain insight into the complex nature of

18 biological models, as well as point to the future goals and difficulties with completely automated 19 model assembly.

\section{Results}

\section{Dataset of True and Candidate Knowledge}


1 We selected a network size of approximately 50 nodes to represent a typical biological model

2 requiring reasonable computational time. We assume that complete models, before any edges are

3 removed represent golden models, ideal representations of the actual system. When creating the

4 golden models, the components, structure, and performance are assumed to be known perfectly

5 (which is most often not the case when using existing knowledge about the biological systems).

6 Once removed from the golden model, we save the edges as candidate knowledge for model

7 assembly. Finally, to account for variable candidate knowledge, we add a list of false edges to

8 the list of true edges from the golden model and use this new set as an input to FIDDLE. We

9 created two different types of random networks, and the descriptive statistics of these networks

10 are shown in Table 1.

11

12 Table 1: Summary statistics of the golden models created as an ER random network or a

13 BA scale-free network structure.

\begin{tabular}{ccc}
\hline Information & ER & BA \\
\hline \hline Number of Nodes & $48.4 \pm 1.4$ & $50.0 \pm 0.0$ \\
Number of Edges & $85.5 \pm 8.9$ & $96.0 \pm 0.0$ \\
Model Density & $0.037 \pm 0.003$ & $0.039 \pm 0.000$ \\
Model Average Degree & $3.53 \pm 0.31$ & $3.84 \pm 0.00$ \\
Undirected Model Clustering & $0.06 \pm 0.03$ & $0.20 \pm 0.06$ \\
Undirected Model Diameter & $6.9 \pm 0.7$ & $5.0 \pm 0.4$ \\
\hline
\end{tabular}

14

15 A summary of the ER random networks and BA networks generated as golden models.

16 Fifty networks were made of each type, and the averages and standard deviations for all

17 network characteristics were determined. 
1 We removed edges from the random networks assuming different probabilities of removal, 0.1 ,

$20.25,0.5,0.75$, or 1 , and stored the removed edges so they could be used by FIDDLE in the

3 model assembly process. Descriptive statistics describing the removal of edges from the 50 ER

4 networks and 50 BA networks are shown in Table 2, where Model Density is defined as the ratio

5 of directed edges to nodes, and Model Degree is the sum of all individual node degrees, divided

6 by the total number of nodes. An example of edge removal is shown in Figure 1.

Table 2: Summary Statistics of Edge Removal

\begin{tabular}{ccccc}
\hline \multicolumn{5}{c}{ Random Edge Removal for ER Networks } \\
\hline \hline $\begin{array}{c}\text { Removal } \\
\text { Probability }\end{array}$ & Model Density & $\begin{array}{c}\text { Model Average } \\
\text { Degree }\end{array}$ & $\begin{array}{c}\text { Number of Removed } \\
\text { Model Edges }\end{array}$ & $\begin{array}{c}\text { Number of } \\
\text { Model Edges }\end{array}$ \\
\hline 0 & $0.037 \pm 0.002$ & $3.52 \pm 0.30$ & $0.0 \pm 0.0$ & $85.5 \pm 8.8$ \\
10 & $0.032 \pm 0.003$ & $3.10 \pm 0.33$ & $10.1 \pm 5.1$ & $75.3 \pm 9.0$ \\
25 & $0.027 \pm 0.003$ & $2.59 \pm 0.29$ & $22.7 \pm 6.9$ & $62.8 \pm 7.7$ \\
50 & $0.018 \pm 0.003$ & $1.70 \pm 0.36$ & $44.0 \pm 7.6$ & $41.4 \pm 9.2$ \\
75 & $0.009 \pm 0.002$ & $0.90 \pm 0.23$ & $63.4 \pm 6.9$ & $22.0 \pm 5.9$ \\
100 & $0.000 \pm 0.000$ & $0.00 \pm 0.00$ & $85.5 \pm 8.8$ & $0.0 \pm 0.0$ \\
\hline \multicolumn{7}{c}{ Random Edge Removal of BA Networks } & \\
\hline \hline Removal & Model Density & Model Average & Number of Removed & Number of \\
\hline 0 & $0.039 \pm 0.000$ & $3.84 \pm 0.00$ & Model Edges & Model Edges \\
\hline 10 & $0.035 \pm 0.002$ & $3.46 \pm 0.18$ & $0.0 \pm 0.0$ & $96.0 \pm 0.0$ \\
25 & $0.029 \pm 0.003$ & $2.87 \pm 0.28$ & $9.4 \pm 4.5$ & $86.6 \pm 4.5$ \\
50 & $0.020 \pm 0.003$ & $1.97 \pm 0.28$ & $24.2 \pm 6.9$ & $71.8 \pm 6.9$ \\
75 & $0.010 \pm 0.002$ & $0.97 \pm 0.21$ & $46.6 \pm 6.9$ & $49.4 \pm 6.9$ \\
100 & $0.000 \pm 0.000$ & $0.00 \pm 0.00$ & $71.6 \pm 5.2$ & $24.4 \pm 5.2$ \\
\hline
\end{tabular}

A summary of the features of the ER random networks and BA networks that were

10 generated as golden models, as well as a summary of the random removal of edges. For

11 each network, edges were removed with different probabilities to determine the smallest

12 network required for reconstruction of the original model. 
1 Figure 2: "Illustration of edge removal with varying probabilities" Example of the process

2 of randomly removing model edges with different probabilities. If an edge is removed with

3 0.1 probability, that edge will also be removed from the network for all higher probabilities

4 of edge removal.

6 Applying a particular edge removal probability on a set of networks results in a varying

7 percentage of missing edges among these networks. With each edge having a 0.1 probability of

8 being removed, there are some networks missing 5\% of their edges, while some are missing up

9 to $15 \%$. As shown in Figure 2, the percentages of removed edges are distributed over most of the

10 range between 0 and 100\% (no missing edges to all missing edges). We show in Figure 3 the

11 edge removal probability plotted against Model Density and the Total Model Error. In Methods,

12 we define Total Model Error (TME) as a measure of the difference between the golden and the

13 candidate model performance.

15 Figure 3: "Illustration of the effect of varying edge removal probability on network

16 properties" Analysis of the effect of different edge Removal Probabilities on Model Density

17 (left) and TME (right).

19 Analysis of FIDDLE Model Extension

20 Next, we attempted to reconstruct the models after different percentages of edges had been

21 removed from them. We will refer to edges that were originally in the golden model, before edge

22 removal, as "true" edges, and to edges that were not in the golden model as "false" edges. To

23 determine the impact of candidate knowledge on the model extension procedure, the extension 
1 method takes as input two lists of candidate edges: an Exclusive list (EXC) which contains only

2 the "true" edges that need to be in the golden model and are necessary for exact model

3 reconstruction, and an Inclusive list (INC) which contains all the edges that are in the EXC, as

4 well as an equal number of "false" edges (randomly generated edges between model nodes that

5 were not in the golden model). The creation of the two lists is meant to reflect the two types of

6 information available to modelers: interactions which are plausible and based on strong literature

7 evidence, usually well known to the scientific community and supported by curated databases,

8 and the information with less evidence, such as the candidate edges that we get from the

9 automated natural language processors. We demonstrate these concepts in Figure 4.

11 Figure 4: "Example of model assembly using the INC List" In this example, we extend a

12 Baseline Model with the INC list of candidate edges and generate the Assembled Model.

13 Based on whether candidate edges are part of the Golden Model, and included in the

14 Assembled Model, we can determine whether the edges represent TP, FP, TN, or FN. We

15 calculate Recall, TNR, FDR, and FOR from the number of TP, FP, TN, and FN in the

16 Assembled Model.

18 To measure each method's recall - the fraction of "true" edges that are selected to extend the 19 model with, given a mix of "true" and "false" edges — we first evaluated both methods using the

20 EXC list to extend baseline models, and then we conducted tests with the INC list. The results of

21 each method are shown in Figure 5A. 
1 Figure 5: "Comparison of performance statistics of model extension" (A) Box plots

2 comparing the quartile ranges of the recall of both algorithms under different conditions.

3 The top row is for models based on the ER network while the bottom row is based on BA

4 networks. The left column is comparing the two algorithms when using only the EXC list,

5 while the right column is comparing the algorithms when using the INC list, with equal

6 number of true and false edges. Statistical significance was determined by $t$-test $(* p<$

$70.05, * * * p<0.001$ ). (B) Box plots comparing the quartile ranges of the TNR, FDR, and

8 FOR of both algorithms under different conditions. The top row is for models based on the

9 ER while the bottom row is based on BA. In all cases, both algorithms were given the INC

10 list, with equal number of true and false edges. Statistical significance was determined by t-

11 test $(* \mathrm{p}<0.05, * * * \mathrm{p}<0.001)$

13 To compare the ability of BFA and DFA to handle true edges and false edges, and to

14 differentiate between them, both methods were given the INC list, and tasked to reconstruct both

15 types of networks, ER and BA networks. We decided to use True Negative Rate (TNR), False

16 Discovery Rate (FDR), and the False Omission Rate (FOR) to quantify the ability of BFA and

17 DFA to classify edges. TNR is also known as Specificity or Selectivity, and it measures the

18 ability to avoid incorporating false edges (the number of false edges not added to the model

19 divided by the total number of false edges). FDR is also known as (1 - Precision) or the Positive

20 Predictive Value (PPV), and is the rate of selecting false edges from all edges (the number of

21 false edges added to the model divided by the total number of edges added to the model). FOR is

22 also known as ( 1 - Negative Predictive Value (NPV) ), and is the rate of true edges not being

23 added to the model (the number of true edges not added to the model divided by the total number 
1 of edges not added to the model). Using these classification statistics, we would want a TNR

2 close to one, and an FDR and FOR close to zero. The calculated rates of TNR, FDR, and FOR,

3 are shown in Figure 5B.

4

5 In addition to model reconstruction, we also evaluated our methods on model creation. BFA and

6 DFA algorithms were tasked with minimizing the TME using only the EXC and INC list of

7 interactions and no baseline model. The recall of both BFA and DFA are shown in Figure 6A. To

8 compare the ability of BFA and DFA to detect false edges, we compared networks created by the

9 two algorithms, with respect to TNR, FDR, and FOR, as shown in Figure 6B.

12 Figure 6: "Comparison of performance statistics of model creation" (A) Box plots

13 comparing the quartile ranges of the recall of both algorithms under different conditions.

14 The top row is for models based on the ER random network while the bottom row is based

15 on BA networks. The left column shows the results obtained when using the EXC list in

16 both algorithms, while the right column shows the results when the algorithms were given

17 the INC list, with equal number of true and false edges. Statistical significance was

18 determined by t-test $(* \mathbf{p}<\mathbf{0 . 0 5}, * * * \mathbf{p}<\mathbf{0 . 0 0 1})$. (B) Box plots comparing the quartile ranges

19 of the TNR, FDR, and FOR of both algorithms under different conditions. The top row is

20 for models based on the ER random network while the bottom row is based on BA

21 networks. In all cases, both algorithms were given the INC list, with equal number of true

22 and false edges. Statistical significance was determined by $\mathbf{t}$-test $(* \mathbf{p}<0.05, * * * \mathbf{p}<0.001)$. 
1 Throughout the process of model creation and extension, we were interested in comparing the

2 methods with respect to the gradient of change in the TME and the number of extensions used to

3 rebuild the model. To examine the difference in performance of the BFA and DFA algorithms on

4 the ER and BA networks, we plotted the TME against the number of edges added back to the

5 model. This plot is shown in top row of Figure 7. To account for networks with larger number of

6 edges added back to the model and ones that started out with higher levels of TME, we

7 normalized the plots to the highest level of TME. This normalized version is shown in the

8 bottom row of Figure 7.

10 Figure 7: "Model performance as edges are added back into the model" In the top row of

11 this figure, we see plots of the model TME ( $y$-axis) with number of edges added ( $x$-axis). In

12 the bottom row, we see the plots normalized to TME.

14 Next, we were also interested in visualizing the assembled network that began as an ER or BA

15 network, and that was extended with the BFA or DFA method with a goal of minimizing the

16 TME. These reconstructed networks are shown in Figure 8.

18 Figure 8: "Examples of model assembly in random and scale-free networks" Examples of 19 BFA- and DFA-based model assembly for: (A) ER network models, and (B) BA network 20 models. 
1 Lastly, we were interested in explaining the difference in performance of the two network types

2 with respect to the TME. In Figure 9, we began with either an ER or BA network, and removed

3 every two-edge combination from the golden model. In this way, we created a set of models

4 similar to the golden model except for a different and unique pair of edges that we removed. We

5 then created a heatmap of the TME for each reduced model, based on which two edges were

6 removed. In cases of the diagonal, only one edge was removed and therefore serves as the golden

7 model with only one edge missing. These heatmaps comparing the TME of ER and BA networks

8 are shown in Figure 9.

10 Figure 9: "Heatmap of network response to combinatorial edge removal"

11 These heatmaps compare the differing responses of ER and BA networks under random

12 edge removals. Columns represent the TME for a model with the same edge missing, while

13 rows also represent the TME for a model with a different edge missing. The diagonal

14 represents models with only one edge missing.

\section{Discussion}

17 Automated model assembly and execution is the goal for systems and computational biologists.

18 In this paper, two novel algorithms for bottom-up model creation were evaluated, and

19 emphasized how complicated the models of biological systems are to create. We used artificial

20 networks with different network structure in order to analyze the BFA and DFA algorithm

21 strengths and weaknesses. The following sections discuss the assembly algorithms, their

22 performance on the artificial models, and future work. 


\section{$1 \quad$ Assembling Models}

2 To investigate the ability of our proposed algorithms to assemble a model with the desired

3 behavior, we selected easily tunable random ER, and scale-free BA networks. Many biological

4 systems have been observed to follow a scale-free power distribution (34). We systematically

5 and randomly removed edges from these networks, which allowed for a varying number of edges

6 to be removed from each network. The density of the network was plotted against edge removal

7 probability, in order to understand how dense these networks were, and how edge removal

8 affected density.

10 In Figure 3, we can see that both ER and BA models responded linearly to the removal

11 probability. The linear line observed in BA networks is a result of the method of network

12 creation. Since BA networks were created with the same number of edges (albeit in different

13 configurations, direction, and regulation), Model Density was then a linear function of removal

14 probability. In ER networks, since the number of edges to begin with were different between

15 different networks, the obtained network population is more diverse, although the networks still

16 respond linearly to the edge removal probability. Interestingly, this linear relationship is not

17 conserved when observing model performance (TME) as a function of edge removal probability.

18 Rather than a strict linear relationship (in which each edge is equally responsible for the same dip

19 in model performance), the effect of different removal probabilities seems to have no linear

20 relationship with the TME metric. It appears that a small number of missing edges can have a

21 strong impact on model performance in some cases, while a large number of missing edges may

22 register a small impact. As we will see later, it is likely that there exist multiple local minima in

23 the model TME state space but which fail to encapsulate the total model information. In this 
1 way, we are able to minimize the model TME and yet the assembled model will have a different

2 structure than the golden model.

4 In a paper discussing networks under random or focused attack, Albert et al. (35) used network

5 diameter to illustrate network failure. They were able to show that with the removal of a small

6 number of edges, the diameter of ER networks reacted differently than that of BA networks.

7 Similarly, our study shows that ER networks respond differently to edge removal than BA.

8 Rather than network diameter, the shortest path between any two nodes, we use the TME metric

9 since our models are small by comparison and our networks are directed. In models that aim to

10 capture biological phenomena, nodes with a high degree of connection dwarf the importance,

11 significance, and effect of other nodes $(36,37)$.

\section{Comparison of the BFA and DFA algorithms}

14 Given that we are using random networks as golden models, the random edge removal makes

15 direct comparison difficult. In order to more reliably compare the two algorithms, we applied

16 them to the exact same baseline model, and we used the same set of candidate edges. We then

17 examined the recall, TNR, FDR, and FOR of the two methods.

19 In Figures 4 through 7, we see that the BFA algorithm performs much better in TNR, while the

20 DFA algorithm performs much better in recall. The higher TNR of the BFA is due to the

21 scanning of all possible edge additions to select an edge that leads to largest TME reduction, and

22 therefore, more selective inclusion of edges and an increased probability of including true edges

23 (if not redundant or initially detrimental). DFA exhibits higher recall and includes any edge that 
1 improves model performance. As true edges are more likely to improve model performance,

2 DFA is able to add more true edges regardless of the magnitude of improvement to model

3 performance.

4

5 The BFS algorithm appears to be a better choice for model assembly when the candidate

6 knowledge is a mix of true and false edges, as it will only incorporate edges vital to the model's

7 performance. On the other hand, the DFA method would be a better choice when correct

8 information is provided, as it performs better at reconstructing the golden model with more true

9 edges. Employing the algorithms in a parallel manner would recommend the best edges that are

10 likely to be in the model and suggest additional edges to explore experimentally. Additionally,

11 the BFA method is easily parallelized for rapid execution on large models.

13 To assess the algorithms, we plotted the TME metric as a function of edges as they were added

14 to the model (Figure 8). The steep decrease early on of the BFA method shows the quick

15 incorporation of highly important edges that drastically reduce TME. DFA does not exhibit the

16 same performance as any improvement is added immediately. Changes in TME are similar for

17 ER and BA networks, although BA networks appear to begin with less TME and require a fewer

18 number of edges to improve model performance when compared to ER networks. This holds

19 across different percentages of missing edges except when all edges are removed. Once all edges

20 are removed, BA networks have a worse change in TME with the number of edges added; this is

21 likely due to BA networks possessing more edges on average (an artifact of the network creation

22 method). In each case, there appear to be a small number of edges which can drastically reduce

23 TME, regardless of the overall percent of edges being removed from the model. This is best seen 
1 in instances of 0.5 and 0.75 removal probability. This trait disappears when there is just too

2 much information (edges) missing from the model for complex regulations and causal

3 relationships to form. In comparing the performance of BFA and DFA when given either EXC or

4 INC list, it is interesting to note that the INC list performs almost identically (or only slightly

5 better) to the EXC list in BFA. This demonstrates that only True edges are likely to improve the

6 model performance and trigger a steep decrease in TME.

8 Automatically Created and Extended Models

9 Next, we were interested in exploring the effect of network structure on each algorithm's

10 performance. As we attempted to reconstruct the golden models with FIDDLE, the BFA and

11 DFA algorithms were successfully able to minimize TME; however, the assembled models were

12 not the same as the golden models (Figure 8). The key reason is that these algorithms function to

13 maximize the performance metric, i.e., minimize TME, while adding the least number of edges.

14 Thus, this approach often results in finding local minima and simplifying the model to its most

15 basic causal interactions that reproduce the expected behavior. We demonstrate some of the

16 limitations of applying a simple error function in Figure 9, where two missing true edges can

17 result in both much better and much worse model performance than either edge individually.

18 There have been similar analyses on the complicating factors of inferring biological networks

19 (38). In a comprehensive analysis of the DREAM3 challenge $(38,39)$, four main errors were

20 found that would drastically reduce the reconstruction method's confidence and directly impact

21 the algorithm's performance: fan-out error, fan-in error, cascade error, and feed-forward loop

22 error. These errors are inherent symptoms of characteristics of the underlying model structure:

23 highly redundant network connectivity and complex network motifs. 
1 In a review by Kitano [2], he argues that robustness is a fundamental feature of evolvable

2 complex systems, necessary for them to be robust to environmental and genetic mutations. $\mathrm{He}$

3 describes four key mechanisms that help ensure robustness: system control, alternative

4 mechanisms, modularity, and decoupling. Both the existence of alternative mechanism and

5 complex system controls make it difficult to understand and model these systems. The presence

6 of alternative mechanisms allows for high levels of redundancy in biological networks while

7 system control requires a complex interplay of multiple interactions where missing a small

8 subset will fundamentally change the dynamics of the model. Luckily, biological modularity may

9 hold the key into how we can best understand and model these systems. In this paper, we focused

10 on singular molecular interactions and adding one at a time. Perhaps through analyzing potential

11 interactions in the context of the system and understanding the modules as a whole, we may

12 make model assembly and extension easier as these modules can be added together.

\section{Implications for Creation and Extension Methods}

15 This paper describes an approach for the automatic assembly of biological models. The efficient

16 incorporation of novel information, especially from machine reading systems, is critical to

17 maintain updated models of biological systems. The analysis conducted in this work also enables

18 comparisons with other algorithms such as genetic (31) and clustering algorithms (30) and future

19 efforts to combine these algorithms into a hybrid approach for model extension. There has been

20 considerable work (30) showing different clustering methods of model elements and these will

21 be explored for model assembly. We plan on continuing this study by adding groups of

22 interactions together, the best number to add together and interrogate the traits that make edges

23 "good" to be added together. This is a complex problem as it not only depends on what type the 
1 edges are and where they are added but how they are integrated into the network. Also, while the

2 FIDDLE algorithms do not depend on the type of update functions used to simulate the model

3 (arithmetic, Boolean, etc.), the type of update function is expected to impact model extension

4 accuracy.

5 Further improvements can be realized using a rigorous method for testing models, such as

6 statistical model checking, which allows for efficient verification of system properties (40).

7 Therefore, model checking can be used to select models that best recapitulate real biological

8 systems by estimating how well models satisfy observed or desired system properties.

\section{Conclusions}

11 We have been able to illustrate the advantages and limitations of utilizing breadth or depth first

12 addition algorithms for assembling biological networks from new candidate knowledge extracted

13 from literature. In general, automated assembly of biological models is challenging due to the

14 inherent redundancy and complexity in biological networks. In this paper, we have shown that

15 BFA has higher TNR and DFA is more sensitive when it comes to assembling biological models.

16 These relative strengths suggest different applications: BFA could be used to extend a model in a

17 more focused way, more likely to include only true biological connections while DFA could be

18 used to suggest many different interactions that could be explored for applicability. In general, it

19 appears that BFA is a better method to use if there is an abundance of inaccurate information,

20 while DFA is a better method for accurate information and assembly of a truly representative

21 model. In the future, we plan on improving our algorithms to further advance the effectiveness of

22 automated model assembly from interactions extracted from literature and public databases. 


\section{$1 \quad$ Methods}

2 The FIDDLE tool can be used as a stand-alone tool, or as a part of a larger framework of tools.

3 In this work, we use the DySE framework (41), to simulate, and analyze the models assembled

4 by FIDDLE. Both FIDDLE and DySE are implemented in Python. In addition, the Python

5 package, NetworkX (42) was used to create all networks, while Graphviz (43) was used to

6 visualize them.

\section{Discrete Models and Simulation}

9 The models that we use in this work are composed of elements representing proteins, genes,

10 chemicals, or biological processes, and directed element interactions. For each model element,

11 we define its influence set, consisting of its positive (activating) and negative (inhibiting)

12 regulators. The model structure is a network $\mathrm{G}(\mathrm{V}, \mathrm{E})$, where $\mathrm{V}$ is a set of nodes representing

13 model elements, and $\mathrm{E}$ is a set of directed edges representing regulatory influences between

14 elements. Here, we assume that each model element can be in one of the three states, OFF (value

15 0), LOW activity (value 1), and HIGH activity (value 2). In general, FIDDLE is not limited to

16 elements with only three states and is applicable to any discrete model. Each model element can

17 have an update rule, which is used to determine element's state given the states of its regulators

18 in the influence set. Update rules that we used in this work are summation functions, where the

19 sum of the state values of negative regulators is subtracted from the sum of the state values of

20 positive regulators, although the FIDDLE algorithms are not dependent on the type of update

21 functions. Together, model elements, element influence sets, the number of values for each

22 element, and element update rules, F, and together comprise an executable model, M(V,E,F). 
1 In this work, we use Discrete, Stochastic, Heterogeneous simulator (DiSH) (44), which allows

2 for simulations of discrete models with various types of update functions, and has several

3 different simulation schemes, including deterministic and stochastic. For the analysis we

4 conducted here, we used a uniform, step-based, random-ordered, sequential update scheme

5 (USB-RSQ), described in detail in (44). It has been shown previously $(44,45)$ that the USB-RSQ

6 simulation scheme is able to recapitulate the intra-cellular network dynamics. DiSH simulates

7 the models starting from an initial state (assigned before simulations), and for a pre-defined

8 number of time steps, $T$ (e.g., when the steady state is reached). Each such simulation run, $r$,

9 yields for every model element $\mathrm{v}_{i} \in \mathrm{V}$, a trajectory of values, $S_{\mathrm{v}_{i}}^{r}=\left(s_{\mathrm{v}_{i}, 1}^{r}, s_{\mathrm{v}_{i}, 2}^{r}, \ldots s_{\mathrm{v}_{i}, T}^{r}\right)$, where

$10 s_{\mathrm{v}_{i}, t}^{r}$ is the state value of element $\mathrm{v}_{i}$ at time point $t(t=1, . ., T)$, within run $r$. Due to the randomness

11 of the update scheme, element trajectories may vary across multiple runs that start with the same

12 initial state. Therefore, for the same time step $t$, following the approach from (44), we average

13 the values $S_{\mathrm{v}_{i}, t}^{r}$ across different runs, to obtain average trajectories for all elements. More

14 formally, we compute an average trajectory of model element $\mathrm{v}_{i}$ as:

$$
\bar{S}_{\mathrm{v}_{i}}=\frac{1}{R} \cdot \sum_{r=1}^{R} S_{\mathrm{v}_{i}}^{r}=\frac{1}{R} \cdot \sum_{r=1}^{R}\left(s_{\mathrm{v}_{i}, 1}^{r}, s_{\mathrm{v}_{i}, 2}^{r}, \ldots s_{\mathrm{v}_{i}, T}^{r}\right)=\left(\bar{s}_{\mathrm{v}_{i}, 1}, \bar{s}_{\mathrm{v}_{i}, 2}, \ldots \bar{s}_{\mathrm{v}_{i}, T}\right)
$$

where $R$ is the overall number of conducted simulation runs. Additionally, we denote the average

17 model state at the final time step $T$ as $Q=\left(\bar{S}_{\mathrm{v}_{1}, T}, \bar{S}_{\mathrm{v}_{2}, T}, \ldots, \bar{S}_{\mathrm{v}_{N}, T}\right)$, where $N$ is the number of

18 elements in the model.

20 In the experiments that we describe here, for all model elements $\mathrm{v}_{i} \in \mathrm{V}$, we assume that their

21 initial state value is $s_{\mathrm{v}_{i}, 1}^{r}=1$, and for each created model, we conducted $R=100$ simulation runs,

22 each with $T=2,500$. Given that the networks we use here are artificial (they do not represent any

23 real biological system), there is no information about initial values that should be used for 
1 elements, and therefore, we used the middle value among the three possible state values, to

2 minimize the bias that the lowest and highest state values can have on model dynamics.

4 Model Performance Metric

5 Model accuracy is usually measured by evaluating the model against a set of ground truths, or

6 expected behaviors, that specify how the model is supposed to respond given a certain initial

7 state and input values. Since our goal is to automatically assemble models, we incorporated

8 within our assembly algorithms a metric that assesses how closely to the specified behavior

9 newly created models perform. Our model performance metric examines the states of all model

10 elements in the last time step $T$ of the average trajectories (usually, a steady state). We denote the

11 expected final state vector of the desired behavior, either observed in a real system or assumed to

12 hold for a golden model, as $Q_{\mathrm{GM}}$. Any model that we assemble is considered a candidate model,

13 and we denote its final state as $Q_{\mathrm{CM}}$. We define Total Model Error (TME) as a measure of the

14 difference between the golden and the candidate model behavior, that is, we compute the

15 absolute distance between vectors $Q_{\mathrm{GM}}$ and $Q_{\mathrm{CM}}$ :

16

$$
\operatorname{TME}\left(Q_{\mathrm{GM}}, Q_{\mathrm{CM}}\right)=\sum_{i=1}^{N}\left|\bar{s}_{\mathrm{v}_{i}, T}^{\mathrm{GM}}-\bar{s}_{\mathrm{v}_{i}, T}^{\mathrm{CM}}\right|
$$

17 where $N$ represents the number of elements in the model.

\section{Model Assembly Methodology}

20 The inputs to FIDDLE include: a baseline model, $\mathrm{M}_{\mathrm{BL}}\left(\mathrm{V}_{\mathrm{BL}}, \mathrm{E}_{\mathrm{BL}}, \mathrm{F}_{\mathrm{BL}}\right)$, to be extended by FIDDLE

21 into candidate models, a set of candidate edges, $\mathrm{E}_{\mathrm{NEW}}$, that are used to extend the baseline

22 model, the expected behavior in the form of the final state values of the golden model, $Q_{\mathrm{GM}}$, and

23 the precomputed TME of the baseline model, $\mathrm{TME}_{\mathrm{BL}}$. 
2 The Breadth First Addition (BFA) procedure starts by evaluating the contribution of each new

3 edge from $\mathrm{E}_{\mathrm{NEW}}$ to decreasing $\mathrm{TME}_{\mathrm{BL}}$, that is, we simulate the model that consists of our original

4 baseline model $\mathrm{M}_{\mathrm{BL}}$ and a selected new edge, and we compute TME of that extended model

5 according to Eq 2. Next, we permanently move, from $E_{N E W}$ to the model, the new edge that leads

6 to the largest decrease in the original $\mathrm{TME}_{\mathrm{BL}}$, and then we repeat the procedure with this new

7 extended model, i.e., similar to what we did with the original model, we evaluate addition of the

8 remaining edges from $\mathrm{E}_{\mathrm{NEW}}$ to this new model by computing their TME values. This process is

9 repeated until we either find the extended model that matches the expected end values of the

10 golden model $\left(Q_{\mathrm{GM}}\right)$, there are no more edges in $\mathrm{E}_{\mathrm{NEW}}$, or no edge can be added to the baseline

11 without increasing the TME. Our BFA algorithm is outlined more formally in Figure 10.

\section{Figure 10: "Pseudocode of the BFA Algorithm"}

14 The basic structure of the BFA algorithm in a pseudocode format.

16 The Depth First Addition (DFA) procedure, similar to the BFA algorithm, starts with

17 evaluation of edges from $\mathrm{E}_{\mathrm{NEw}}$ by computing their contribution to decreasing TME of the

18 baseline model. Different from BFA, as soon as we find an edge which leads to a TME lower

19 than the $\mathrm{TME}_{\mathrm{BL}}$, we add that edge to the baseline model, and remove it from $\mathrm{E}_{\mathrm{NEW}}$. We repeat

20 this procedure using the new extended model and the remaining edges from $\mathrm{E}_{\mathrm{NEW}}$, that is, as

21 soon as we encounter an edge that leads to a lower TME than the current model, we add that

22 edge to the model. The DFA algorithm stops when we either find the extended model that

23 matches the expected end values of the golden model $\left(Q_{\mathrm{GM}}\right)$, there are no more edges in $\mathrm{E}_{\mathrm{NEW}}$, or 
1 no edge can be added to the baseline without increasing the TME. Our DFA algorithm is

2 outlined more formally in Figure 11.

4 Figure 11: "Pseudocode of the DFA Algorithm"

5 The basic structure of the DFA algorithm in a pseudocode format.

\section{Evaluation Procedure}

8 To evaluate how well FIDDLE can assemble models and to compare and contrast the BFA and

9 the DFA approach, we apply a method commonly used for model reconstruction (46-50). The

10 first step of this method is a generation of golden models. We used two different types of random

11 networks to create our golden models: one type based on Erdös-Rényi random directed networks

12 (33), and another using the Barabási-Albert scale-free network (34). To generate an Erdös-Rényi

13 Network (ER), a random network was seeded with 50 nodes and a probability of connection of

14 0.035. To create a Barabási-Albert scale-free network (BA), each network was seeded with fifty

15 nodes and two initial edges. For both the ER and BA networks, these parameters were chosen to

16 create artificial networks of roughly the same number of nodes, edges, and density (as shown in

17 Table 1). Models were kept around 50 nodes to best replicate the synthetic models used in the

18 DREAM3 challenge (39). We used the Python library NetworkX (42) to generate these networks

19 and to calculate the network statistics reported in Table 1. Next, we assigned each directed edge

20 in these networks, randomly with equal likelihood, either a positive or negative sign (activating

21 or inhibiting, respectively). Finally, we simulated golden models to record the $Q_{\mathrm{GM}}$ values, which

22 are used by the TME function to guide the BFA and DFA algorithms. 
1 In the second step of the evaluation procedure, we created baseline models by randomly

2 removing edges from the golden models. Specifically, we select the removal probability to be

$30.1,0.25,0.5,0.75$, or 1 , and for each edge in the model we randomly choose whether to remove

4 it or not with that selected probability. For example, in a model with 0.5 edge removal

5 probability, an edge was selected, given a 50-50 chance of being removed, either removed or not,

6 and then the next edge was selected. If an edge was removed in a model of low removal

7 probability, it was also missing from a model of higher removal probability. The removed edges

8 were stored in a list called the Exclusive list (EXC) since it only contains edges that were

9 removed from the golden model. We also create an Inclusive list (INC), which contains every

10 edge in EXC, and an equal number of false edges that are not present in the golden model. The

11 INC and EXC lists are used in the evaluation procedure, where they serve as the candidate

12 knowledge (edges) input to FIDDLE.

14 We conducted exhaustive analysis of all possible combinations of baseline model (with $0.1,0.25$,

$150.5,0.75$, or 1 probability of removing edges from the golden model), extension algorithm (BFA

16 or DFA), and candidate edges (EXC or INC list). In each experiment, we used the final state of

17 the golden model, $Q_{\mathrm{GM}}$, when computing the model performance metric TME of assembled

18 candidate models, in an effort to reconstruct the golden model, or assemble a model that

19 reproduces the same final state as the golden model.

\section{List of Abbreviations}

22 BFA: Breadth First Addition

23 DFA: Depth First Addition 
1 ER: Erdös-Rényi Random Network

2 EXC: Exclusive List

3 FOR: False Omission Rate

4 FDR: False Discovery Rate

5 INC: Inclusive List

6 BA: Barabási-Albert Scale-Free Network

$7 \quad$ Declarations

8 Acknowledgments

9 The authors would like to thank Kai-wen Liang for his instrumental work in the implementation

10 of the BFA algorithm.

\section{Authors' contributions}

$12 \mathrm{AB}$ conceived the project. $\mathrm{AB}$ created the BFA and DFA algorithms. AB generated all of the

13 networks, the simulation of models, and the analysis of the results. AB, NM, and CT interpreted

14 the results and wrote the manuscript. All authors read and approved the final manuscript.

15 Availability of data and materials

16 - Project name: FIDDLE

17 - Project home page: https://bitbucket.org/biodesignlab/fiddle/

18 - Archived version: 2c6ad05

19 - Operating system(s): Linux/Mac

20 - Programming language: Python3

25 Consent for publication

26 Not applicable.

\section{Competing Interests}

28 The authors declare that they have no competing interests. 


\section{Ethics approval and consent to participate}

2 Not applicable.

\section{$3 \quad$ Funding}

4 This work was funded in part by DARPA award W911NF-17-1-0135.

1. Epstein JM. Why Model? 2008.

2. Sobie EA, Lee Y-S, Jenkins SL, Iyengar R. Systems biology—biomedical modeling. Sci Signal. 2011;4(190):tr2-tr.

3. Schäfer J, Strimmer K. An empirical Bayes approach to inferring large-scale gene association networks. Bioinformatics. 2004;21(6):754-64.

4. Küffner R, Petri T, Tavakkolkhah P, Windhager L, Zimmer R. Inferring gene regulatory networks by ANOVA. Bioinformatics. 2012;28(10):1376-82.

5. Raza K. Fuzzy logic based approaches for gene regulatory network inference. Artificial intelligence in medicine. 2018.

6. Madhamshettiwar PB, Maetschke SR, Davis MJ, Reverter A, Ragan MA. Gene regulatory network inference: evaluation and application to ovarian cancer allows the prioritization of drug targets. Genome medicine. 2012;4(5):41.

7. D'haeseleer P, Liang S, Somogyi R. Genetic network inference: from co-expression clustering to reverse engineering. Bioinformatics. 2000;16(8):707-26.

8. Linde J, Schulze S, Henkel SG, Guthke R. Data-and knowledge-based modeling of gene regulatory networks: an update. EXCLI journal. 2015;14:346.

9. Wani N, Raza K. Integrative Approaches to Reconstruct Regulatory Networks From MultiOmics Data: A Review of State-of-the-Art Methods. 2018.

10. Hecker M, Lambeck S, Toepfer S, Van Someren E, Guthke R. Gene regulatory network inference: data integration in dynamic models—a review. Biosystems. 2009;96(1):86-103.

11. Banf M, Rhee SY. Enhancing gene regulatory network inference through data integration with markov random fields. Scientific reports. 2017;7:41174.

12. Recamonde-Mendoza M, Werhli AV, Biolo A. Systems biology approach identifies key regulators and the interplay between miRNAs and transcription factors for pathological cardiac hypertrophy. Gene. 2019.

13. Fabregat A, Jupe S, Matthews L, Sidiropoulos K, Gillespie M, Garapati P, et al. The Reactome Pathway Knowledgebase. Nucleic Acids Research. 2018;46(D1):D649-D55.

14. Karp PD, Riley M, Paley SM, Pellegrini-Toole A. The MetaCyc Database. Nucleic acids research. 2002;30(1):59-61.

15. Türei D, Korcsmáros T, Saez-Rodriguez J. OmniPath: guidelines and gateway for literaturecurated signaling pathway resources. Nature Methods. 2016;13(12):966-7.

16. Szklarczyk D, Morris JH, Cook H, Kuhn M, Wyder S, Simonovic M, et al. The STRING database in 2017: quality-controlled protein-protein association networks, made broadly accessible. Nucleic acids research. 2017;45(D1):D362-D8. 
17. Schaefer CF, Anthony K, Krupa S, Buchoff J, Day M, Hannay T, et al. PID: the pathway interaction database. Nucleic Acid Res. 2009;37.

18. Slenter DN, Kutmon M, Hanspers K, Riutta A, Windsor J, Nunes N, et al. WikiPathways: a multifaceted pathway database bridging metabolomics to other omics research. Nucleic acids research. 2018;46(D1):D661-D7.

19. Chelliah V, Juty N, Ajmera I, Ali R, Dumousseau M, Glont M, et al. BioModels: ten-year anniversary. Nucleic Acids Research. 2015;43(D1):D542-D8.

20. Helikar T, Kowal B, McClenathan S, Bruckner M, Rowley T, Madrahimov A, et al. The Cell Collective: toward an open and collaborative approach to systems biology. BMC Syst Biol. 2012;6.

21. Kanehisa M, Furumichi M, Tanabe M, Sato Y, Morishima K. KEGG: new perspectives on genomes, pathways, diseases and drugs. Nucleic Acids Research. 2017;45(D1):D353-D61.

22. Valenzuela-Escárcega MA, Hahn-Powell G, Surdeanu M. Description of the Odin Event Extraction Framework and Rule Language. 2015.

23. Ferguson G, Allen JF, editors. TRIPS: An integrated intelligent problem-solving assistant 1998: AAAI Press.

24. Hakala K, Van Landeghem S, Salakoski T, Van de Peer Y, Ginter F. Application of the EVEX resource to event extraction and network construction: Shared Task entry and result analysis. BMC Bioinformatics. 2015;16(Suppl 16):S3-S.

25. Burns GAPC, Dasigi P, de Waard A, Hovy EH. Automated detection of discourse segment and experimental types from the text of cancer pathway results sections. Database : the journal of biological databases and curation. 2016;2016.

26. Buchel F, Rodriguez N, Swainston N, Wrzodek C, Czauderna T, Keller R, et al. Path2Models: large-scale generation of computational models from biochemical pathway maps. BMC Syst Biol. 2013;7(1):116.

27. Gyori BM, Bachman JA, Subramanian K, Muhlich JL, Galescu L, Sorger PK. From word models to executable models of signaling networks using automated assembly. Molecular systems biology. 2017;13(11):954-.

28. Sharp R, Pyarelal A, Gyori B, Alcock K, Laparra E, Valenzuela-Escárcega MA, et al., editors. Eidos, INDRA, \& Delphi: From free text to executable causal models. Proceedings of the 2019 Conference of the North American Chapter of the Association for Computational Linguistics (Demonstrations); 2019.

29. Liang K-W, Wang Q, Telmer C, Ravichandran D, Spirtes P, Miskov-Zivanov N. Methods to Expand Cell Signaling Models Using Automated Reading and Model Checking. Springer, Cham; 2017. p. 145-59.

30. Ahmed Y, Telmer C, Miskov-Zivanov N. ACCORDION: Clustering and Selecting Relevant Data for Guided Network Extension and Query Answering. arXiv preprint arXiv:200205748. 2020.

31. Sayed K, Bocan KN, Miskov-Zivanov N, editors. Automated Extension of Cell Signaling Models with Genetic Algorithm2018/07//: IEEE.

32. Kozen DC. Depth-First and Breadth-First Search. The Design and Analysis of Algorithms. New York, NY: Springer New York; 1992. p. 19-24.

33. Erdős P, Rényi A. ON THE EVOLUTION OF RANDOM GRAPHS.

34. Barabasi A-L, Albert R. Emergence of scaling in random networks. Science (New York, NY). 1999;286(5439):509-12. 
35. Albert R, Jeong H, Barabási A-L. Error and attack tolerance of complex networks. Nature. 2000;406(6794):378-82.

36. Crucitti P, Latora V, Marchiori M, Rapisarda A. Efficiency of scale-free networks: error and attack tolerance. Physica A: Statistical Mechanics and its Applications. 2003;320:622-42.

37. Jeong H, Mason SP, Barabási A-L, Oltvai ZN. Lethality and centrality in protein networks. Nature. 2001;411(6833):41.

38. Marbach D, Prill RJ, Schaffter T, Mattiussi C, Floreano D, Stolovitzky G. Revealing strengths and weaknesses of methods for gene network inference. Proceedings of the national academy of sciences. 2010;107(14):6286-91.

39. Prill RJ, Marbach D, Saez-Rodriguez J, Sorger PK, Alexopoulos LG, Xue X, et al. Towards a rigorous assessment of systems biology models: the DREAM3 challenges. PloS one. 2010;5(2):e9202.

40. Legay A, Delahaye B, Bensalem S. Statistical Model Checking: An Overview. Springer, Berlin, Heidelberg; 2010. p. 122-35.

41. Telmer C, Sayed K, Butchy AA, Bocan KN, Holtzapple E, Hansen CE, et al. Dynamic System Explanation, DySE, a framework that evolves to reason about complex systems. Artificial Intelligence for Data Discovery and Reuse. 2019.

42. Hagberg A, Schult D, Swart P. Proceedings of the Python in Science Conference (SciPy): Exploring Network Structure, Dynamics, and Function using NetworkX. 2008. p. 11-5.

43. Ellson J, Gansner ER, Koutsofios E, North SC, Woodhull G. Graphviz and dynagraphstatic and dynamic graph drawing tools. Graph drawing software: Springer; 2004. p. 127-48.

44. Sayed K, Kuo Y-H, Kulkarni A, Miskov-Zivanov N. Dish simulator: capturing dynamics of cellular signaling with heterogeneous knowledge. Proceedings of the 2017 Winter Simulation Conference; Las Vegas, Nevada. 3242250: IEEE Press; 2017. p. 1-12.

45. Assmann SM, Albert R. Discrete Dynamic Modeling with Asynchronous Update, or How to Model Complex Systems in the Absence of Quantitative Information. In: Belostotsky DA, editor. Plant Systems Biology. Totowa, NJ: Humana Press; 2009. p. 207-25.

46. Marbach D, Schaffter T, Mattiussi C, Floreano D. Generating realistic in silico gene networks for performance assessment of reverse engineering methods. Journal of computational biology. 2009;16(2):229-39.

47. Ghanbari M, Lasserre J, Vingron M. Reconstruction of gene networks using prior knowledge. BMC systems biology. 2015;9(1):84.

48. Mendes P, Sha W, Ye K. Artificial gene networks for objective comparison of analysis algorithms. Bioinformatics. 2003;19(suppl_2):ii122-ii9.

49. Muldoon JJ, Yu JS, Fassia M-K, Bagheri N. Network inference performance complexity: a consequence of topological, experimental and algorithmic determinants. Bioinformatics. 2019.

50. Van den Bulcke T, Van Leemput K, Naudts B, van Remortel P, Ma H, Verschoren A, et al. SynTReN: a generator of synthetic gene expression data for design and analysis of structure learning algorithms. BMC bioinformatics. 2006;7(1):43. 
Figures

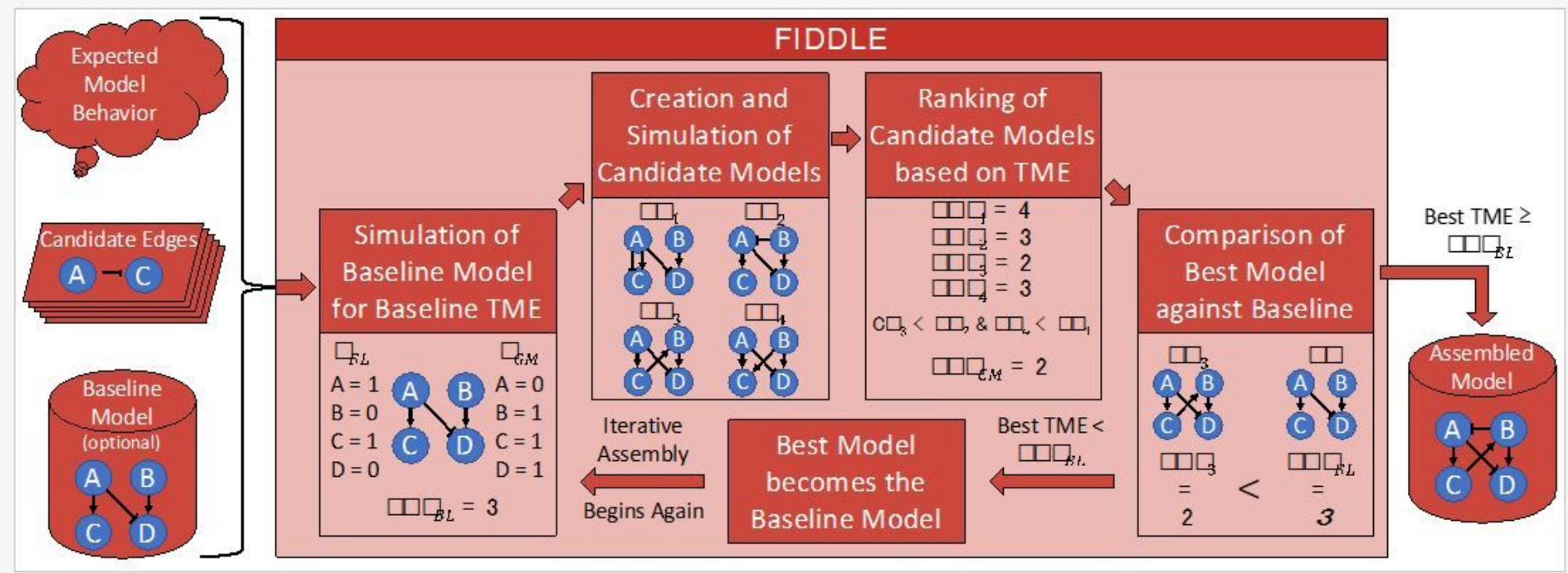

Figure 1

"Overview of the FIDDLE process" FIDDLE is a robust model assembly tool which requires candidate edges, an expectation of model behavior, and an optional baseline model. FIDDLE iteratively assembles candidate edges into candidate models, simulates them, and calculates their performance using the TME metric.

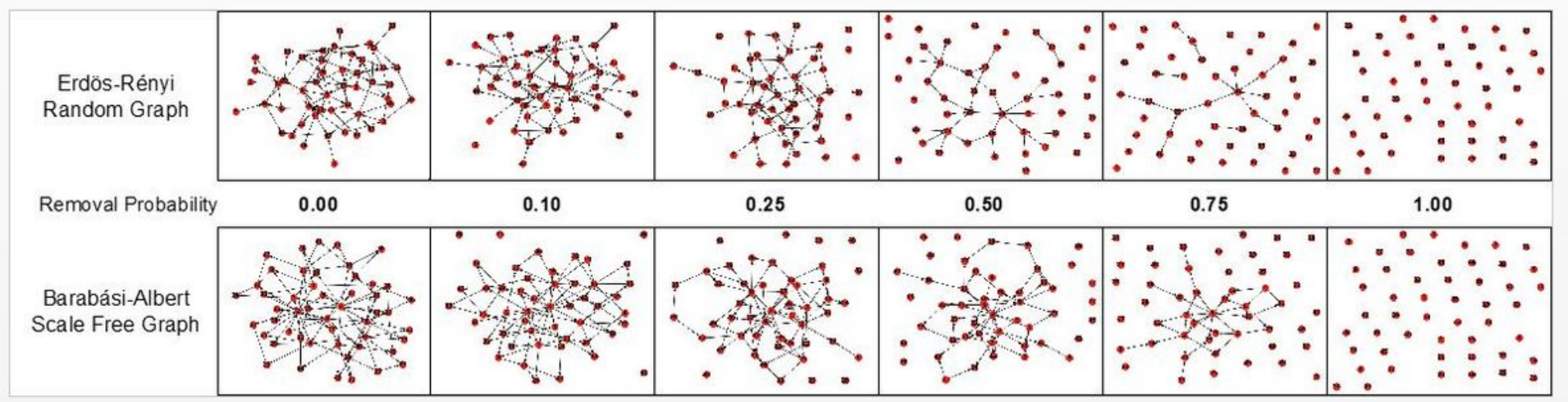

Figure 2

"Illustration of edge removal with varying probabilities" Example of the process of randomly removing model edges with different probabilities. If an edge is removed with 0.1 probability, that edge will also be removed from the network for all higher probabilities of edge removal. 

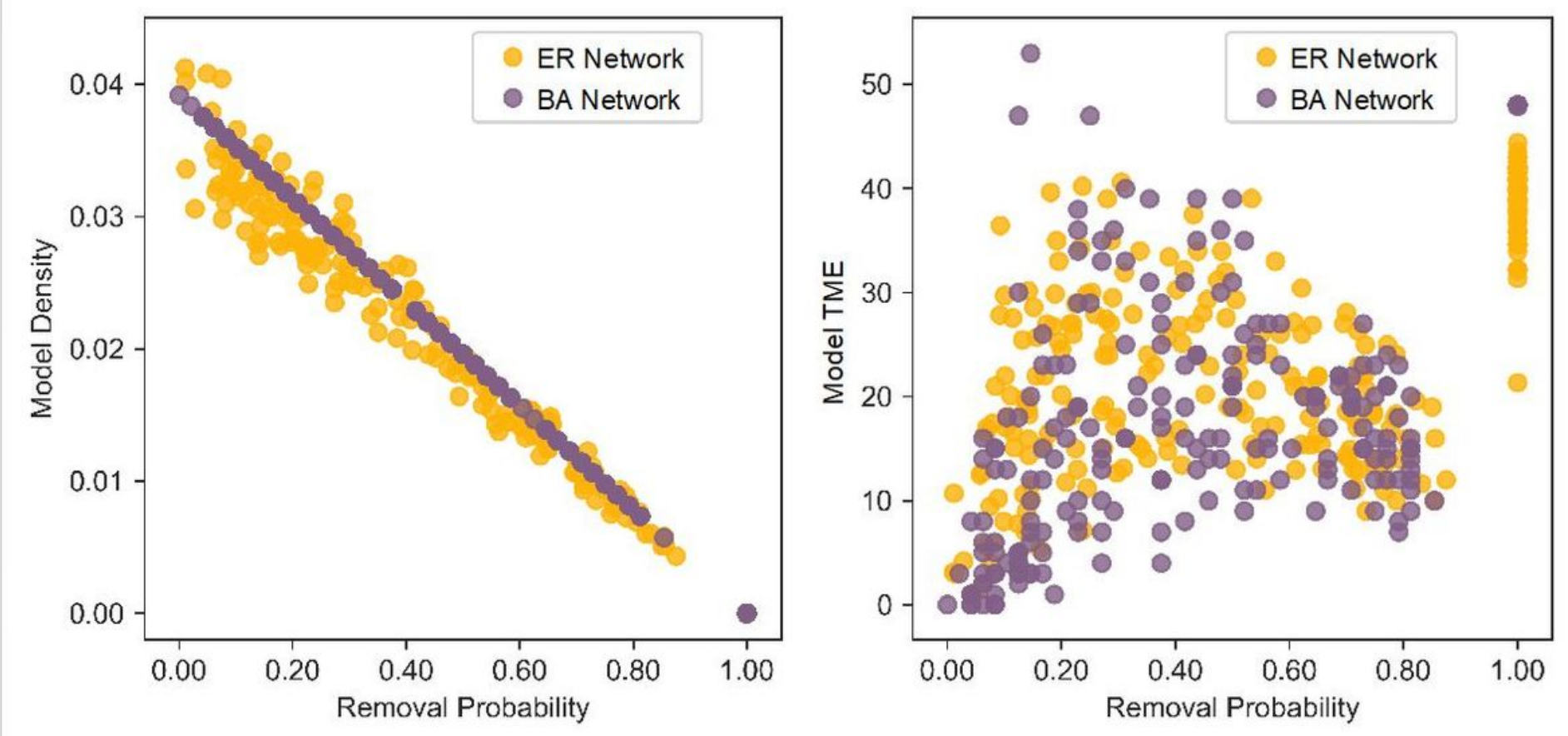

\section{Figure 3}

"Illustration of the effect of varying edge removal probability on network properties" Analysis of the effect of different edge Removal Probabilities on Model Density (left) and TME (right).

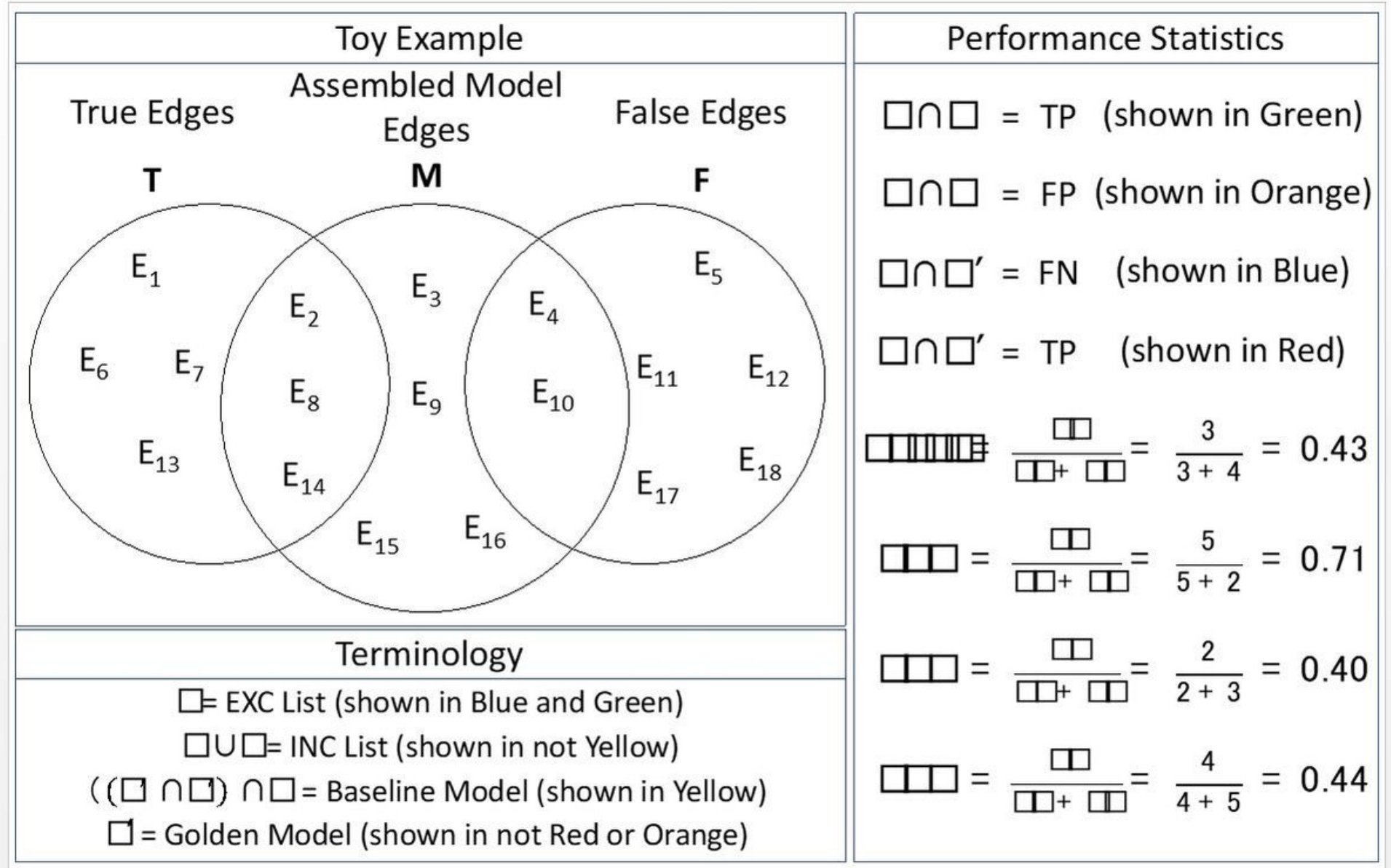


Figure 4

"Example of model assembly using the INC List" In this example, we extend a Baseline Model with the INC list of candidate edges and generate the Assembled Model. Based on whether candidate edges are part of the Golden Model, and included in the Assembled Model, we can determine whether the edges represent TP, FP, TN, or FN. We calculate Recall, TNR, FDR, and FOR from the number of TP, FP, TN, and FN in the Assembled Model.

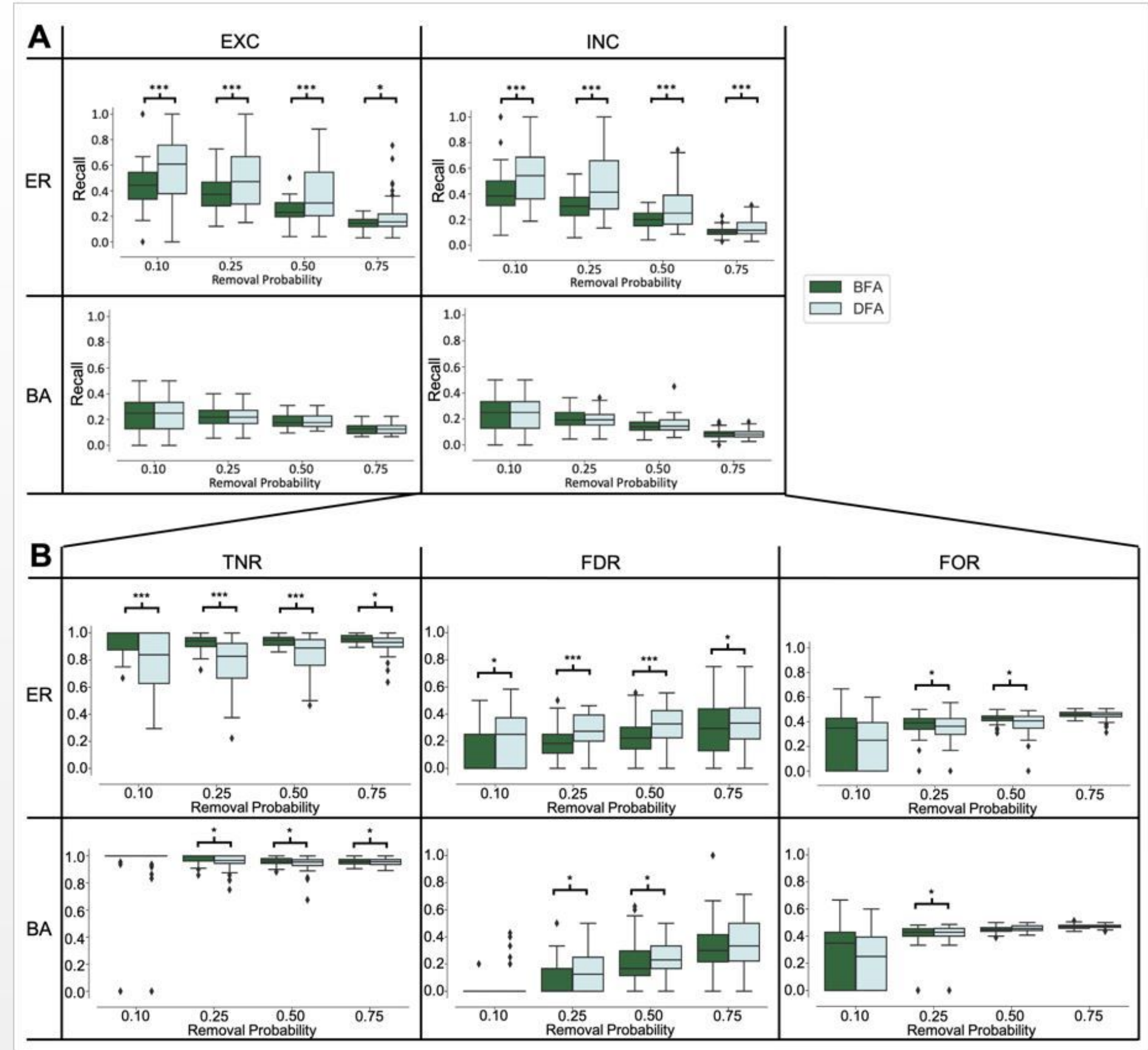

Figure 5

"Comparison of performance statistics of model extension" (A) Box plots comparing the quartile ranges of the recall of both algorithms under different conditions. The top row is for models based on the ER 
network while the bottom row is based on BA networks. The left column is comparing the two algorithms when using only the EXC list, while the right column is comparing the algorithms when using the INC list, with equal number of true and false edges. Statistical significance was determined by t-test $(* p<0.05$, $\star \star \star x<0.001)$. (B) Box plots comparing the quartile ranges of the TNR, FDR, and FOR of both algorithms under different conditions. The top row is for models based on the ER while the bottom row is based on BA. In all cases, both algorithms were given the INC list, with equal number of true and false edges. Statistical significance was determined by t-test $(* p<0.05$, $* \star \star ~ p<0.001)$.

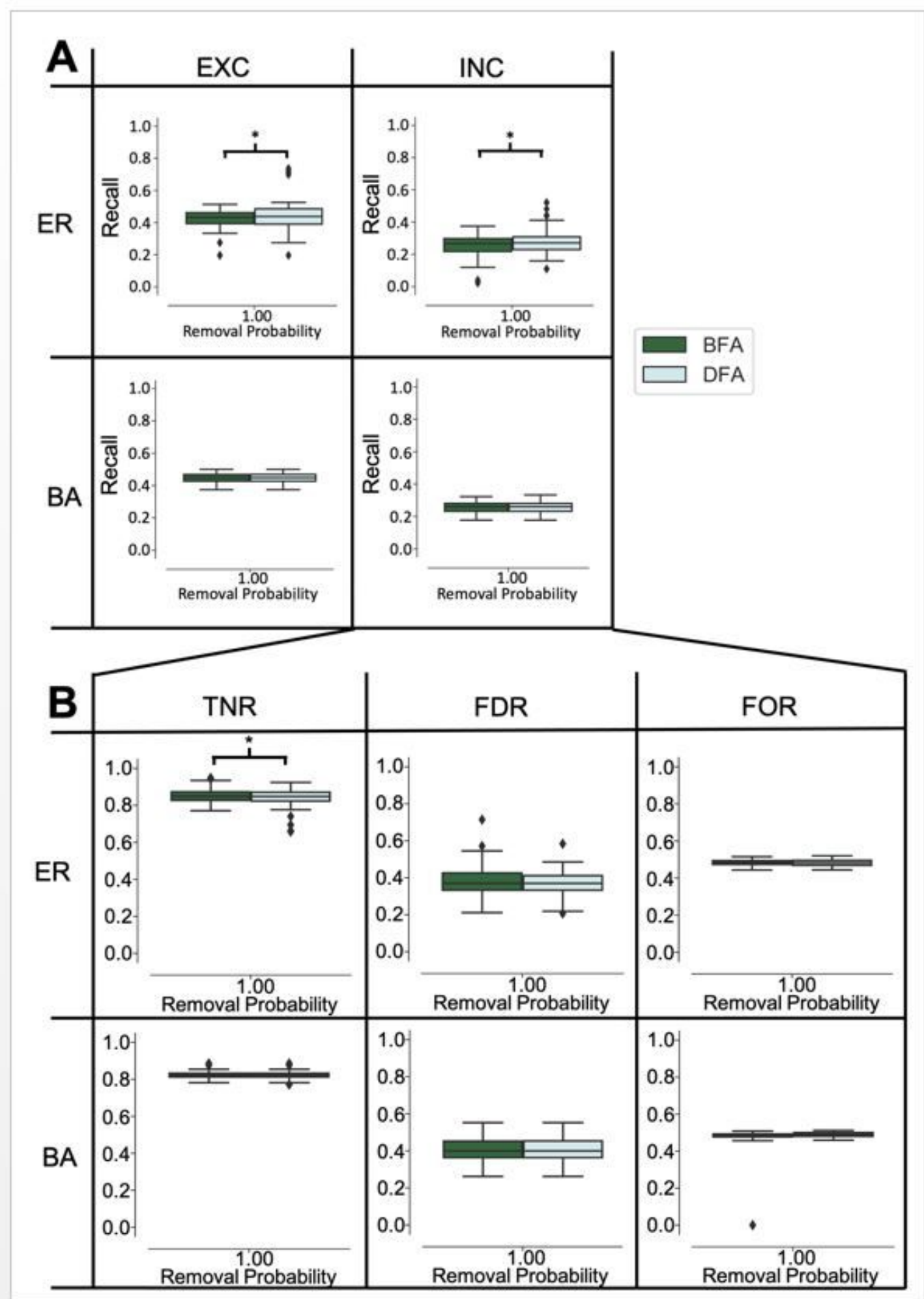




\section{Figure 6}

"Comparison of performance statistics of model creation" (A) Box plots comparing the quartile ranges of the recall of both algorithms under different conditions. The top row is for models based on the ER random network while the bottom row is based on BA networks. The left column shows the results obtained when using the EXC list in both algorithms, while the right column shows the results when the algorithms were given the INC list, with equal number of true and false edges. Statistical significance was determined by t-test ( ${ }^{*} p<0.05$, $\left.* \star * p<0.001\right)$. (B) Box plots comparing the quartile ranges of the TNR, FDR, and FOR of both algorithms under different conditions. The top row is for models based on the ER random network while the bottom row is based on BA networks. In all cases, both algorithms were given the INC list, with equal number of true and false edges. Statistical significance was determined by t-test (* $p<0.05, * * *<<0.001)$.

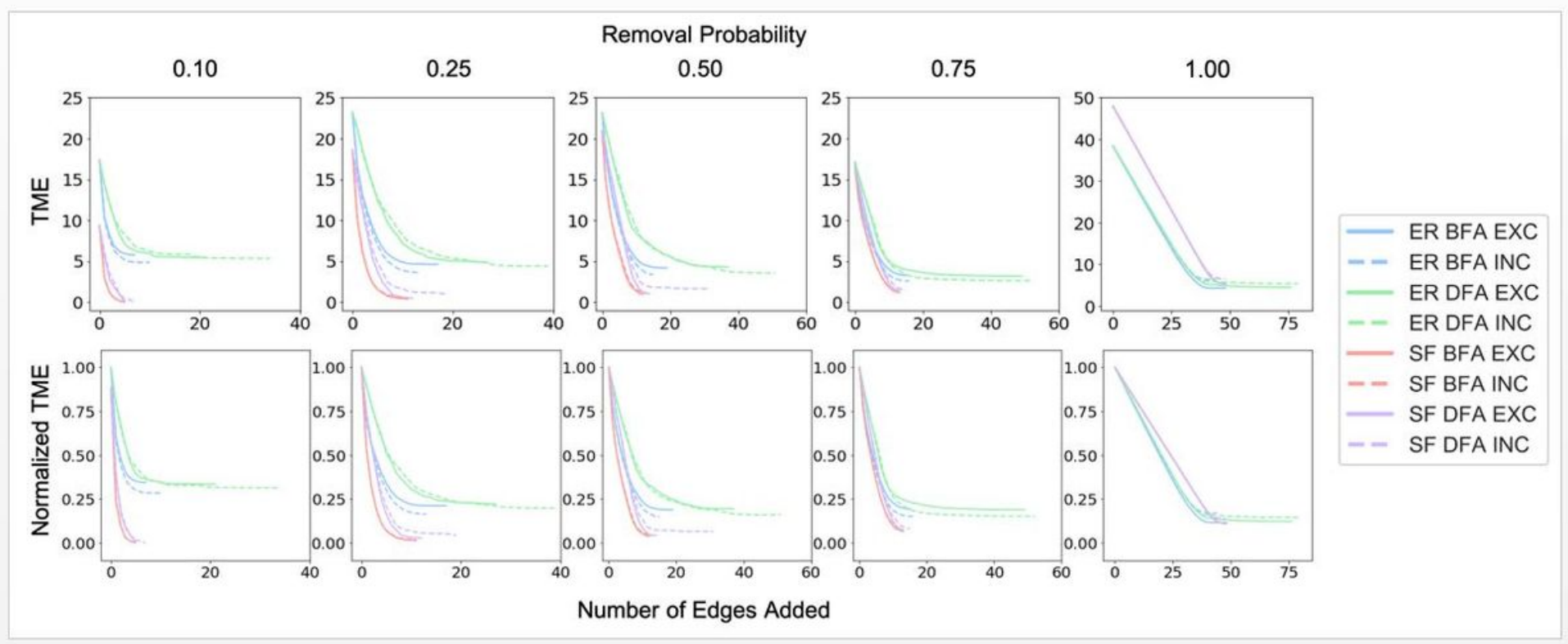

\section{Figure 7}

"Model performance as edges are added back into the model" In the top row of this figure, we see plots of the model TME (y-axis) with number of edges added (x-axis). In the bottom row, we see the plots normalized to TME. 


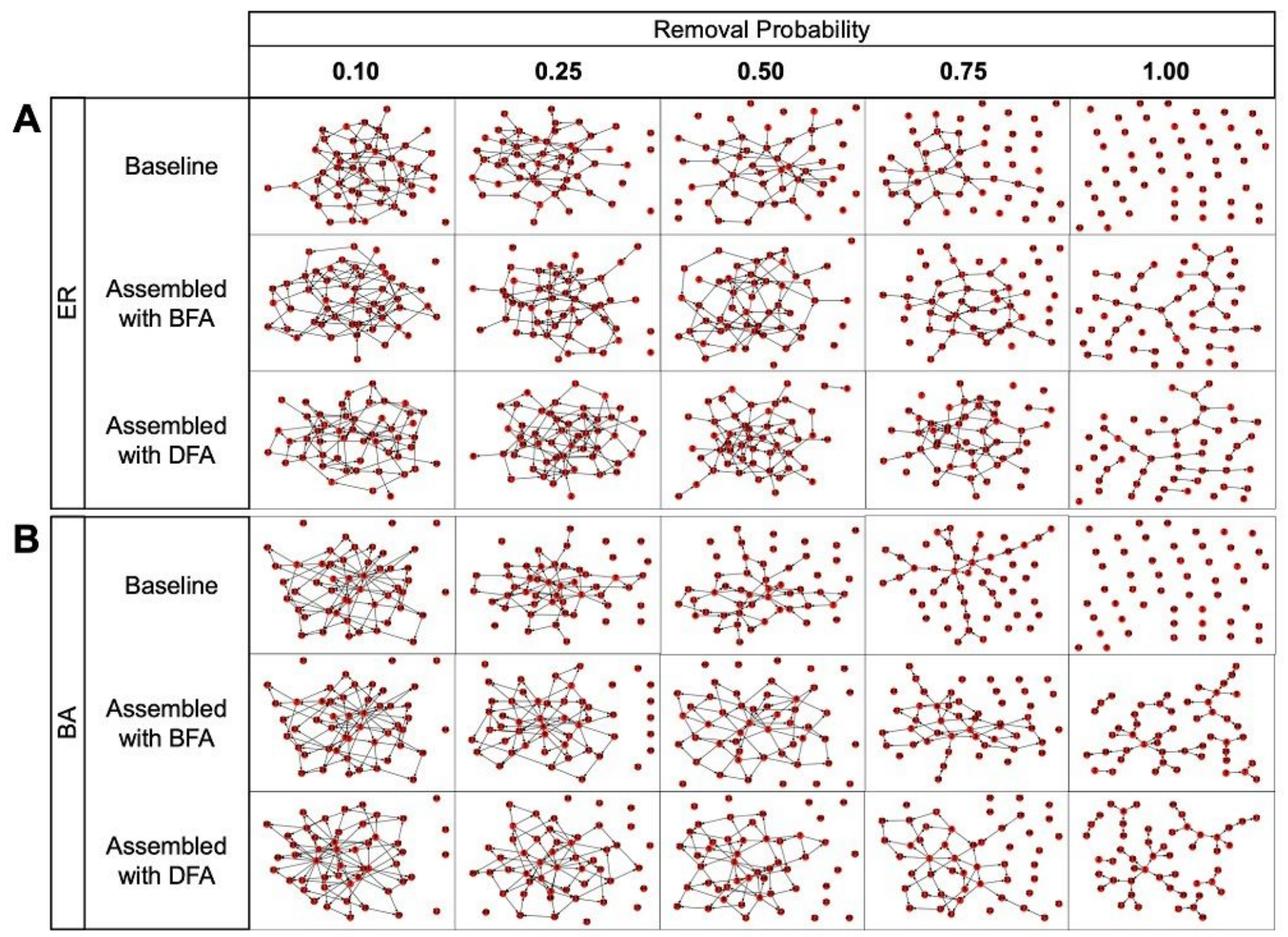

Figure 8

"Examples of model assembly in random and scale-free networks" Examples of BFA- and DFA-based model assembly for: (A) ER network models, and (B) BA network models. 


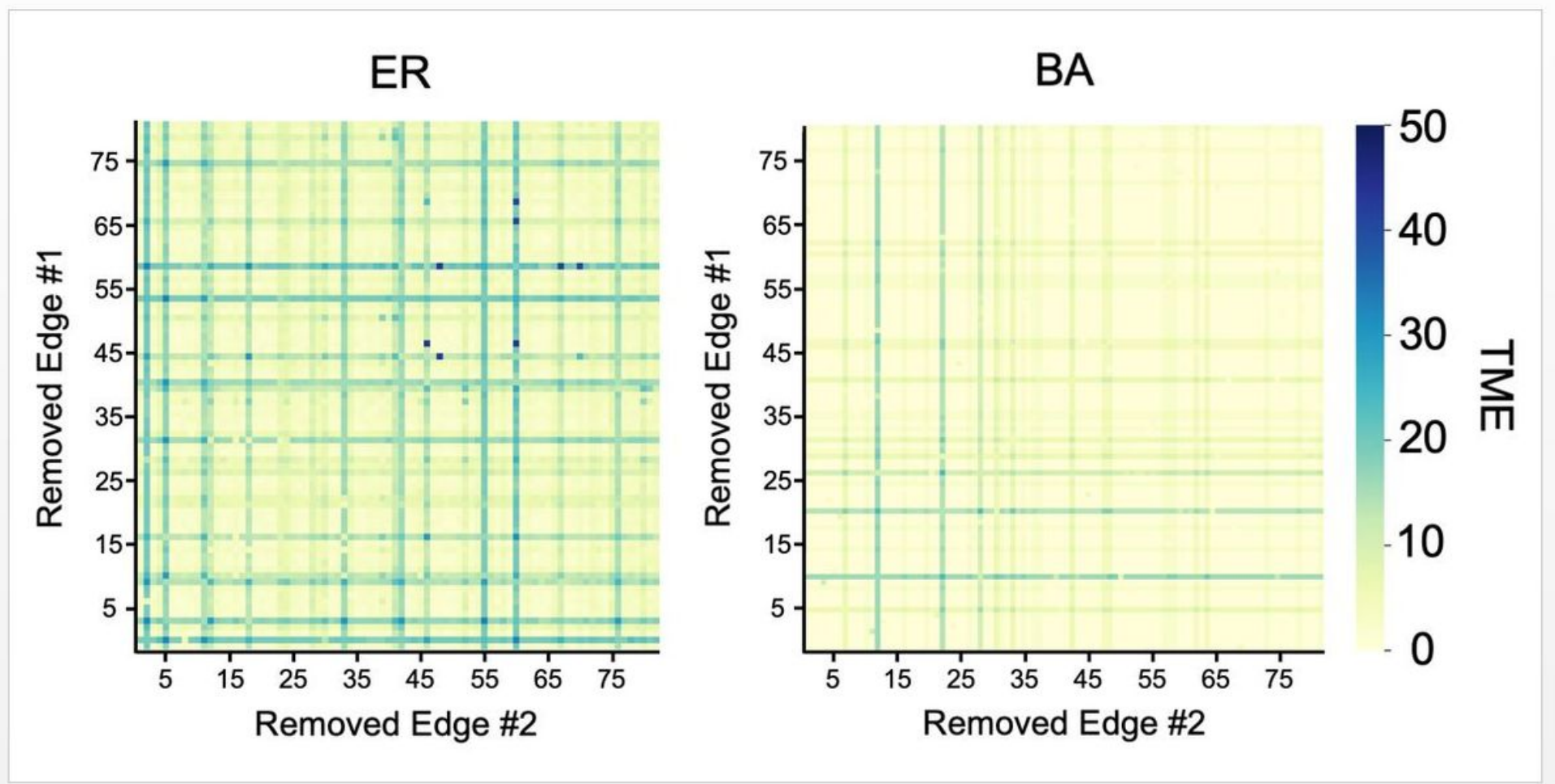

Figure 9

"Heatmap of network response to combinatorial edge removal" These heatmaps compare the differing responses of ER and BA networks under random edge removals. Columns represent the TME for a model with the same edge missing, while rows also represent the TME for a model with a different edge missing. The diagonal represents models with only one edge missing. 


\section{Algorithm: Breadth First Addition (BFA)}

Input: baseline model $\left(\mathrm{M}_{\mathrm{BL}}\right)$, list of edges $\left(\mathrm{E}_{\mathrm{NEW}}\right)$, $\mathrm{TME}$ of the baseline model $\left(\mathrm{TME}_{\mathrm{BL}}\right)$, expected performance of the golden model $\left(Q_{\mathrm{GM}}\right)$,

Output: extended baseline model that minimizes the TME

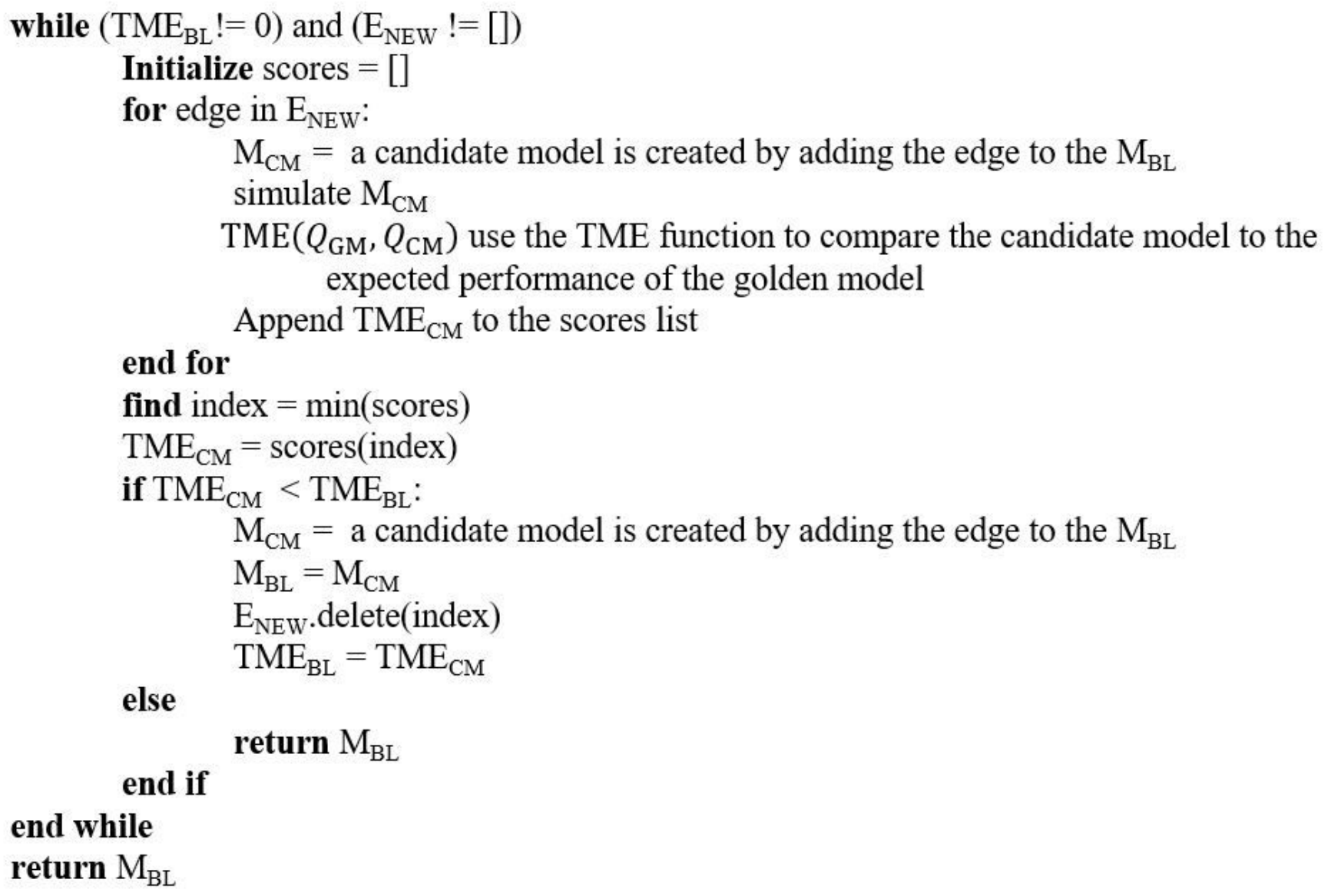

Figure 10

"Pseudocode of the BFA Algorithm" The basic structure of the BFA algorithm in a pseudocode format. 


\section{Algorithm: Depth First Addition (DFA)}

Input: baseline model, list of edges, expected performance of the golden model, current TME Output: extended baseline model that minimizes the TME

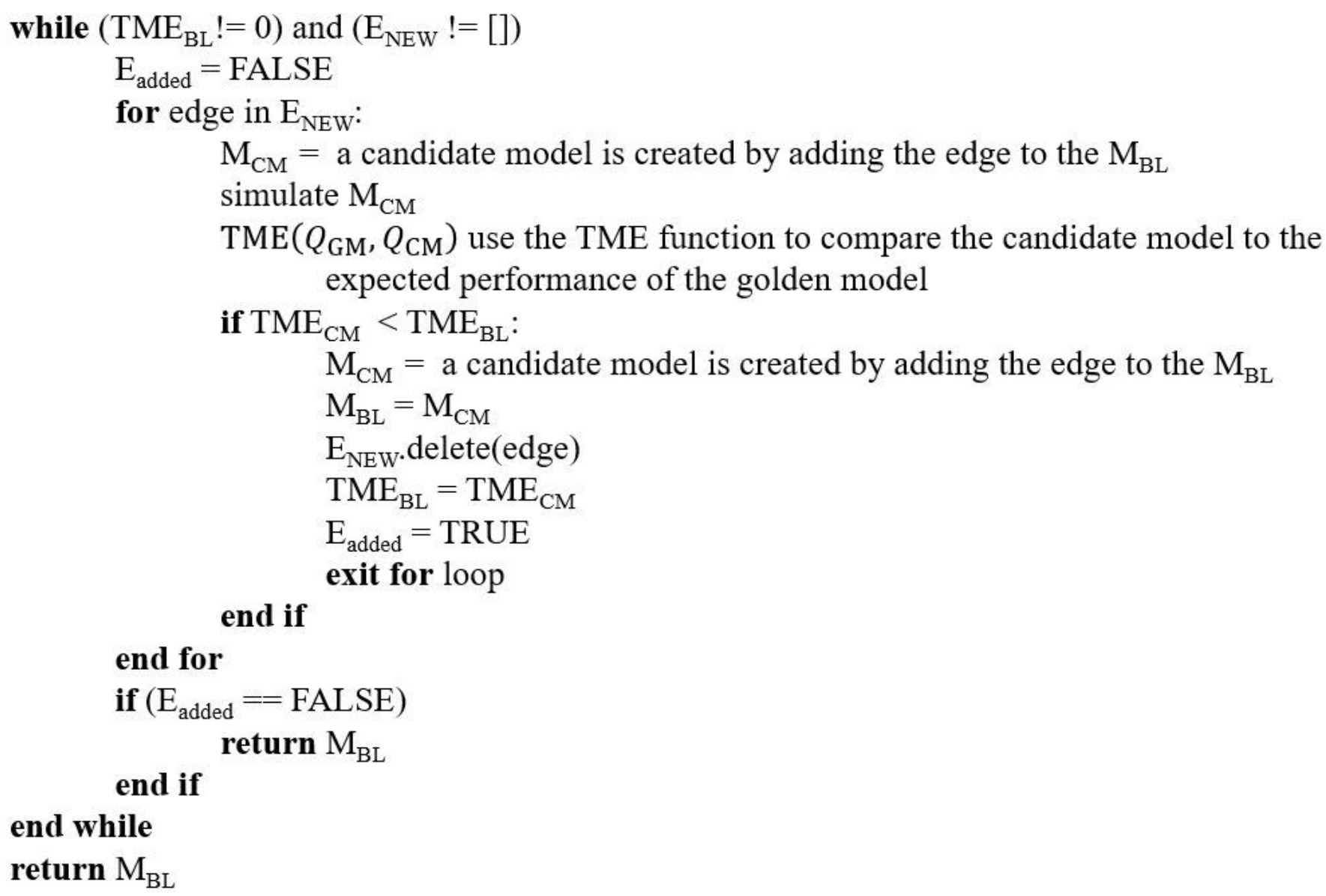

\section{Figure 11}

"Pseudocode of the DFA Algorithm" The basic structure of the DFA algorithm in a pseudocode format. 